\title{
Restricted Use Pesticides 1
}

\section{Frederick M. Fishel $^{2}$}

Restricted use pesticides (RUP) are only for retail sale to, and use by, certified applicators or persons under their direct supervision, and only for those purposes covered by the applicator's certification. When a pesticide is classified as restricted, the label will state "Restricted Use Pesticide" at the top of the front panel. Below this heading may be a statement describing the reason for the restricted classification. There are various reasons which are determined by the U.S. Environmental Protection Agency (EPA) why a particular pesticide is classified as restricted. These criteria involve the EPA's determination that the pesticide may be hazardous to human health or to the environment even when used according to the label.

Some states, including Florida, adhere to the EPA's federal listing for determining those pesticide active ingredients that are classified as "restricted." Other states may require that certain other active ingredients not listed on the federal list be classified as "restricted" in their states due to local conditions, generally related to environmental concerns.

Reasons for RUP classification that are given in this guide may include terminology such as:
- Fetotoxicity - adverse effects on the fetus;

- Mutagenicity - causes genetic changes in the organism which may or may not be passed on to its next generation;

- Oncogenicity - causes tumors, but not necessarily malignant and

- Teratogenicity - causes birth defects.

The pesticide's label is the last word on use, regardless of its classification as a general use or a restricted use pesticide.

Table 1 provides a listing of common names of pesticides determined to be classified as restricted use by the EPA. With each entry, formulations that are regarded as restricted, their uses and the reason(s), if given, for their restricted classification are cited. This listing does not contain common names of pesticides that have had their registrations cancelled or those whose restricted status is no longer in effect, nor is there a listing of trade names. Be aware that the listing is slightly modified, generally on an annual basis. For updated listings of federal restricted use pesticides, see: http://www.epa.gov/opprd001/rup.

1. This document is PI-36, one of a series of the Pesticide Information Office, Florida Cooperative Extension Service, Institute of Food and Agricultural Sciences, University of Florida. Published March 2005. Visit the EDIS Web Site at http://edis.ifas.ufl.edu.

2. Frederick M. Fishel, Associate Professor, Agronomy Department, and Director, Pesticide Information Office; Florida Cooperative Extension Service, Institute of Food and Agricultural Sciences, University of Florida, Gainesville, FL 32611. 
Table 1. Restricted use pesticides.

\begin{tabular}{|c|c|c|c|}
\hline $\begin{array}{l}\text { Pesticide common } \\
\text { name }\end{array}$ & Specific formulations & Specific uses & Criteria for RUP \\
\hline Acetochlor & Emulsifiable concentrate & $\begin{array}{l}\text { Field corn, popcorn, } \\
\text { forage/feeder corn }\end{array}$ & $\begin{array}{l}\text { Ground and surface water } \\
\text { concerns }\end{array}$ \\
\hline Acrolein & As sole active ingredient & All uses & $\begin{array}{l}\text { Human inhalation hazard, } \\
\text { adverse effects on avian } \\
\text { and aquatic organisms }\end{array}$ \\
\hline Alachlor & All formulations & All uses & Oncogenic potential \\
\hline Aldicarb & $\begin{array}{l}\text { As sole active ingredient } \\
\text { and in combination with } \\
\text { other actives; all granular } \\
\text { formulations }\end{array}$ & All uses & Accident history \\
\hline Aluminum phosphide & As sole active ingredient & All uses & Human inhalation hazard \\
\hline Amitraz & All formulations & Pears & Oncogenic potential \\
\hline Amitrole & All formulations & All uses except homeowner & Oncogenic potential \\
\hline Arsenic acide & $\begin{array}{l}\text { All formulations except } \\
\text { brush-on }\end{array}$ & $\begin{array}{l}\text { All dessicant uses; all wood } \\
\text { preservative uses }\end{array}$ & $\begin{array}{l}\text { Oncogenicity, } \\
\text { mutagenicity and } \\
\text { repro/fetotoxicity }\end{array}$ \\
\hline Arsenic pentoxide & All formulations & Wood preservative uses & $\begin{array}{l}\text { Potential oncogenicity, } \\
\text { mutagenicity, } \\
\text { repro/fetotoxicity }\end{array}$ \\
\hline Atrazine & $\begin{array}{l}\text { All manufacturing and } \\
\text { end use }\end{array}$ & $\begin{array}{l}\text { Agricultural and industrial } \\
\text { uses }\end{array}$ & $\begin{array}{l}\text { Ground water } \\
\text { contamination potential; } \\
\text { worker exposure concerns }\end{array}$ \\
\hline Avermectin & Emulsifiable concentrate & Cotton and citrus & $\begin{array}{l}\text { Toxic to fish, mammals } \\
\text { and aquatic organisms }\end{array}$ \\
\hline Avitrol & All formulations & All uses & $\begin{array}{l}\text { Hazard to fish and } \\
\text { non-target birds }\end{array}$ \\
\hline Azinphos-methyl & $\begin{array}{l}\text { All liquids with greater } \\
\text { than } 13.5 \% \\
\text { concentration; others on } \\
\text { a case-by-case basis }\end{array}$ & All uses & $\begin{array}{l}\text { Human inhalation hazard, } \\
\text { acute toxicity, hazard to } \\
\text { avian, aquatic and } \\
\text { mammalian species }\end{array}$ \\
\hline Bendiocarb & $\begin{array}{l}\text { Granular and wettable } \\
\text { powder }\end{array}$ & Turf & $\begin{array}{l}\text { Toxicity to aquatic and } \\
\text { avian species }\end{array}$ \\
\hline Biphenthrin & Emulsifiable concentrate & Cotton & $\begin{array}{l}\text { Toxic to fish and aquatic } \\
\text { organisms }\end{array}$ \\
\hline Bis(tributyltin)oxide & Solution - ready to use & Antifouling paint & $\begin{array}{l}\text { Toxic to aquatic } \\
\text { organisms including } \\
\text { shellfish }\end{array}$ \\
\hline Carbofuran & $\begin{array}{l}\text { All formulations except } \\
\text { pellets and tablets }\end{array}$ & All uses & $\begin{array}{l}\text { Acute inhalation toxicity; } \\
\text { avian toxicity (granular) }\end{array}$ \\
\hline Chlorethoxyfos & Granular & $\begin{array}{l}\text { Corn: field, forage, pop, } \\
\text { sweet }\end{array}$ & $\begin{array}{l}\text { Acute human, avian and } \\
\text { aquatic invertebrate toxicity }\end{array}$ \\
\hline Chlorophacinone & $\begin{array}{l}\text { Tracking powder, dust } \\
\text { and ready to use } 0.2 \% \\
\text { (EPA Reg. Nos. } \\
7173-113 \text { and } 7173-172\end{array}$ & Inside buildings & $\begin{array}{l}\text { Human hazard, potential } \\
\text { for food contamination, } \\
\text { possible inhalation hazard }\end{array}$ \\
\hline Chloropicrin & $\begin{array}{l}\text { All formulations greater } \\
\text { than } 2 \% \text { and all } \\
\text { formulations (rodent } \\
\text { control) }\end{array}$ & $\begin{array}{l}\text { All uses (greater than } 2 \% \\
\text { including rodent control) }\end{array}$ & $\begin{array}{l}\text { Acute inhalation toxicity, } \\
\text { hazard to non-target } \\
\text { organisms }\end{array}$ \\
\hline
\end{tabular}


Table 1. Restricted use pesticides.

\begin{tabular}{|c|c|c|c|}
\hline $\begin{array}{l}\text { Pesticide common } \\
\text { name }\end{array}$ & Specific formulations & Specific uses & Criteria for RUP \\
\hline Chlorpyrifos & $\begin{array}{l}\text { Emulsifiable } \\
\text { concentrate }\end{array}$ & Wheat & Avian and aquatic toxicity \\
\hline Chromic acid & $\begin{array}{l}\text { All formulations except } \\
\text { brush-on }\end{array}$ & All wood preservative & $\begin{array}{l}\text { Oncogenicity, } \\
\text { mutagenicity, } \\
\text { teratogenicity and } \\
\text { fetotoxic effects } \\
\end{array}$ \\
\hline Clofentezine & All formulations & All uses & $\begin{array}{l}\text { Additional data required to } \\
\text { remove the restriction }\end{array}$ \\
\hline Coal tar & Solution - ready to use & Wood preservative & $\begin{array}{l}\text { Oncogenicity and } \\
\text { mutagenicity }\end{array}$ \\
\hline Coal tar creosote & All formulations & Wood preservative & $\begin{array}{l}\text { Possible oncogenic and } \\
\text { mutagenic effects }\end{array}$ \\
\hline Coumaphos & Flowable concentrate & $\begin{array}{l}\text { Indoor food and indoor } \\
\text { nonfood }\end{array}$ & $\begin{array}{l}\text { Acute oral toxicity } \\
\text { hazards }\end{array}$ \\
\hline Creosote oil & Al formulations & Wood preservative & $\begin{array}{l}\text { Possible oncogenic and } \\
\text { mutagenic effects }\end{array}$ \\
\hline $\begin{array}{l}\text { Cube resins other than } \\
\text { Rote PM }\end{array}$ & $\begin{array}{l}\text { Emulsifiable } \\
\text { concentrate }\end{array}$ & $\begin{array}{l}\text { Small fruits, currants, } \\
\text { certain berries }\end{array}$ & $\begin{array}{l}\text { Chronic eye and inhalation } \\
\text { effects }\end{array}$ \\
\hline Cuprous oxide & Ready to use & Certain berries & Not specified \\
\hline Cyfluthrin & $\begin{array}{l}25 \% \text { Emulsifiable } \\
\text { concentrate }\end{array}$ & Agricultural & $\begin{array}{l}\text { Acute toxicity to } \\
\text { applicators, fish and other } \\
\text { aquatic organisms }\end{array}$ \\
\hline Cyhalothrin & $\begin{array}{l}\text { Emulsifiable } \\
\text { concentrate }\end{array}$ & Cotton & $\begin{array}{l}\text { Environmental data } \\
\text { requirements }\end{array}$ \\
\hline Cypermethrin & All formulations & All agricultural crops & $\begin{array}{l}\text { Oncogenicity, hazard to } \\
\text { non-target organisms }\end{array}$ \\
\hline Deltamethrin & $\begin{array}{l}\text { Emulsifiable } \\
\text { concentrate }\end{array}$ & Cotton & $\begin{array}{l}\text { High toxicity to aquatic } \\
\text { organisms }\end{array}$ \\
\hline Diazinon & $\begin{array}{l}\text { Granular, emulsifiable } \\
\text { concentrate and wettable } \\
\text { powders }\end{array}$ & $\begin{array}{l}\text { Small fruits and certain } \\
\text { berries }\end{array}$ & Avian and aquatic toxicity \\
\hline Dichlobenil & 2,6-dichlorobenzonitrile & Terrestrial & Conditional \\
\hline Dichloropropene & $\begin{array}{l}\text { All formulations ( } 94 \% \\
\text { liquid concentrate is the } \\
\text { only formulation) }\end{array}$ & All uses & $\begin{array}{l}\text { Probable human } \\
\text { carcinogen, oncogenic, } \\
\text { acutely toxic by oral and } \\
\text { inhalation routes }\end{array}$ \\
\hline Diclofop methyl & All formulations & All uses & Oncogenicity \\
\hline Dicrotophos & $\begin{array}{l}\text { All liquid formulations } 8 \% \\
\text { and greater }\end{array}$ & All uses & $\begin{array}{l}\text { Acute dermal toxicity, } \\
\text { residue effects on avian } \\
\text { species }\end{array}$ \\
\hline Diflubenzuron & Wettable powders & All uses & Hazard to wildlife \\
\hline Dioxathion & $\begin{array}{l}\text { All concentrate solutions } \\
\text { or emulsifiable } \\
\text { concentrates greater } \\
\text { than } 30 \% \text {; all solutions } \\
3 \% \text { and greater for } \\
\text { domestic uses }\end{array}$ & All uses & Acute dermal toxicity \\
\hline
\end{tabular}


Table 1. Restricted use pesticides.

\begin{tabular}{|c|c|c|c|}
\hline $\begin{array}{l}\text { Pesticide common } \\
\text { name }\end{array}$ & Specific formulations & Specific uses & Criteria for RUP \\
\hline Disulfoton & $\begin{array}{l}\text { All ECs } 65 \% \text { and greater, } \\
\text { all ECs and concentrate } \\
\text { solutions } 21 \% \text { and } \\
\text { greater with fensulfothion } \\
43 \% \text { and greater, all ECs } \\
32 \% \text { and greater in } \\
\text { combination with } 32 \% \\
\text { fensulfothion and } \\
\text { greater }\end{array}$ & $\begin{array}{l}\text { All uses, commercial seed } \\
\text { treatment (non-aqueous } \\
\text { solution } 95 \% \text { and greater). }\end{array}$ & $\begin{array}{l}\text { Acute dermal toxicity, } \\
\text { inhalation hazard }\end{array}$ \\
\hline Emamectin benzoate & $\begin{array}{l}\text { 4-epimethlyamino-4- } \\
\text { deoxykavermectin BLA } \\
\text { and B1b benzoates }\end{array}$ & Insecticide, miticide & Toxicity to fish \\
\hline Endrin & $\begin{array}{l}\text { 9.4\% liquid (all others } \\
\text { cancelled) }\end{array}$ & Bird perch use & $\begin{array}{l}\text { Acute dermal toxicity, } \\
\text { hazard to non-target } \\
\text { organisms }\end{array}$ \\
\hline Ethion & Only two products & All uses & Acute toxicity \\
\hline Ethoprop & $\begin{array}{l}\text { Emulsifiable } \\
\text { concentrates } 40 \% \text { and } \\
\text { greater (aquatic uses); all } \\
\text { uses (granular and } \\
\text { fertilizer formulations }\end{array}$ & $\begin{array}{l}\text { Aquatic uses (ECs } 40 \% \text { or } \\
\text { greater); all uses (granular } \\
\text { and fertilizer formulations) }\end{array}$ & Acute dermal toxicity \\
\hline Ethyl parathion & All formulations & All uses & $\begin{array}{l}\text { Inhalation hazard to } \\
\text { humans, acute dermal } \\
\text { toxicity, residue effects on } \\
\text { mammalian, aquatic and } \\
\text { avian species, accident } \\
\text { history }\end{array}$ \\
\hline Fenamiphos & $\begin{array}{l}\text { Emulsifiable } \\
\text { concentrates } 35 \% \text { and } \\
\text { greater }\end{array}$ & All uses & $\begin{array}{l}\text { Acute dermal toxicity, } \\
\text { avian acute oral toxicity, } \\
\text { acute inhalation toxicity }\end{array}$ \\
\hline Fenbutatin-oxide & Wettable powder & Grapes & $\begin{array}{l}\text { Very high toxicity to } \\
\text { aquatic organisms }\end{array}$ \\
\hline Fenitrothion & $\begin{array}{l}\text { Emulsifiable concentrate, } \\
93 \% \text { soluble } \\
\text { concentrate/liquid }\end{array}$ & Only forestry uses & $\begin{array}{l}\text { Potential adverse effects } \\
\text { on aquatic and avian } \\
\text { species }\end{array}$ \\
\hline Fenpropathrin & $\begin{array}{l}2.4 \text { emulsifiable } \\
\text { concentrate spray }\end{array}$ & Cotton & $\begin{array}{l}\text { Environmental concerns: } \\
\text { toxic to fish and aquatic } \\
\text { organisms }\end{array}$ \\
\hline Fenthion & $\begin{array}{l}\text { Emulsifiable } \\
\text { concentrate }\end{array}$ & Mosquitocide & $\begin{array}{l}\text { Very high acute toxicity to } \\
\text { birds, fish and aquatic } \\
\text { invertebrates }\end{array}$ \\
\hline Fenvalerate & $\begin{array}{l}\text { Emulsifiable } \\
\text { concentrates }(30 \%)\end{array}$ & Outdoor uses & $\begin{array}{l}\text { Possible adverse effects } \\
\text { on aquatic organisms }\end{array}$ \\
\hline Fipronil & All formulations & Insecticide/miticide & Conditional amended \\
\hline Hydrogen cyanamide & $50 \%$ active ingredient & Desert grown grapes & $\begin{array}{l}\text { Corrosive effects to skin } \\
\text { and eyes }\end{array}$ \\
\hline Lambda-cyhalothrin & All formulations & All uses & $\begin{array}{l}\text { Toxicity to fish and aquatic } \\
\text { invertebrates }\end{array}$ \\
\hline
\end{tabular}


Table 1. Restricted use pesticides.

\begin{tabular}{|c|c|c|c|}
\hline $\begin{array}{l}\text { Pesticide common } \\
\text { name }\end{array}$ & Specific formulations & Specific uses & Criteria for RUP \\
\hline Lindane & $\begin{array}{l}\text { All formulations for } \\
\text { various uses }\end{array}$ & $\begin{array}{l}\text { Commercial ornamentals, } \\
\text { pecans, avocados, } \\
\text { livestock, forestry, } \\
\text { Christmas trees, dog } \\
\text { dusts/shampoos and } \\
\text { structural treatment }\end{array}$ & Possibly oncogenic \\
\hline $\begin{array}{l}\text { Magnesium } \\
\text { phosphide }\end{array}$ & All formulations & All uses & Inhalation hazard \\
\hline Methamidophos & $\begin{array}{l}\text { Liquid formulations } 40 \% \\
\text { and greater, dust } \\
\text { formulations } 2.5 \% \text { and } \\
\text { greater }\end{array}$ & All uses & $\begin{array}{l}\text { Acute dermal toxicity, } \\
\text { residue effects on avian } \\
\text { species }\end{array}$ \\
\hline Methidathion & All formulations & $\begin{array}{l}\text { All uses except nursery } \\
\text { stock, safflower and } \\
\text { sunflower }\end{array}$ & $\begin{array}{l}\text { Residue effects on avian } \\
\text { species }\end{array}$ \\
\hline Methiocarb & All formulations & $\begin{array}{l}\text { Outdoor commercial and } \\
\text { agricultural uses }\end{array}$ & $\begin{array}{l}\text { Possible hazard to avian, } \\
\text { fish and other aquatic } \\
\text { organisms }\end{array}$ \\
\hline Methomyl & $\begin{array}{l}\text { As sole active ingredient } \\
\text { in } 1 \text { to } 2.5 \% \text { baits (except } \\
1 \% \text { fly bait), all } \\
\text { concentrate solution } \\
\text { formulations and } 90 \% \\
\text { wettable powder } \\
\text { formulations (not in water } \\
\text { soluble bags) }\end{array}$ & $\begin{array}{l}\text { Nondomestic outdoor and } \\
\text { all other registered uses } \\
\text { (agricultural crops, } \\
\text { ornamentals and turf) }\end{array}$ & $\begin{array}{l}\text { Residue effects on } \\
\text { mammalian species, other } \\
\text { hazards - accident } \\
\text { history }\end{array}$ \\
\hline Methyl bromide & All formulations & All uses & $\begin{array}{l}\text { Acute toxicity and accident } \\
\text { history }\end{array}$ \\
\hline Methyl isothiocyanate & Solution - ready to use & $\begin{array}{l}\text { Fungicide for wood, wood } \\
\text { preservative }\end{array}$ & $\begin{array}{l}\text { Exceeds classification } \\
\text { criteria of } 40 \text { CFR } \\
152.170\end{array}$ \\
\hline Methyl parathion & All formulations & All uses & $\begin{array}{l}\text { Residue effects on } \\
\text { mammalian and avian } \\
\text { species, hazard to bees, } \\
\text { acute dermal toxicity }\end{array}$ \\
\hline Mevinphos & $\begin{array}{l}\text { All emulsifiable } \\
\text { concentrates and liquid } \\
\text { concentrates - psycodid } \\
\text { filter fly liquid } \\
\text { formulations }-2 \% \text { dust }\end{array}$ & All uses & $\begin{array}{l}\text { Acute dermal toxicity, } \\
\text { residue effects on } \\
\text { mammalian and avian } \\
\text { species }\end{array}$ \\
\hline Niclosamide & $\begin{array}{l}\text { All wettable powders } \\
70 \% \text { and greater }\end{array}$ & All uses & $\begin{array}{l}\text { Acute inhalation toxicity, } \\
\text { effects on aquatic } \\
\text { organisms }\end{array}$ \\
\hline Nicotine & $\begin{array}{l}\text { Liquid and dry } \\
\text { formulations } 14 \% \text { and } \\
\text { greater (greenhouse); all } \\
\text { formulations } \\
\text { (cranberries) }\end{array}$ & $\begin{array}{l}\text { Greenhouse applications, } \\
\text { all applications to } \\
\text { cranberries }\end{array}$ & $\begin{array}{l}\text { Acute inhalation toxicity, } \\
\text { effects on aquatic } \\
\text { organisms }\end{array}$ \\
\hline Nitrogen, liquid & Solution - ready to use & Termiticide & $\begin{array}{l}\text { Highly corrosive upon } \\
\text { contact with skin or eyes }\end{array}$ \\
\hline
\end{tabular}


Table 1. Restricted use pesticides.

\begin{tabular}{|c|c|c|c|}
\hline $\begin{array}{l}\text { Pesticide common } \\
\text { name }\end{array}$ & Specific formulations & Specific uses & Criteria for RUP \\
\hline Oxamyl & $\begin{array}{l}\text { Liquid formulations, } \\
\text { granular on a } \\
\text { case-by-case basis }\end{array}$ & All uses & $\begin{array}{l}\text { Acute oral toxicity, acute } \\
\text { inhalation toxicity, avian } \\
\text { oral toxicity }\end{array}$ \\
\hline Oxydemeton methyl & All products & All uses & Reproductive effects \\
\hline Paraquat & $\begin{array}{l}\text { All formulations and } \\
\text { concentrations except } \\
\text { certain mixtures - see } \\
\text { label }\end{array}$ & All uses & $\begin{array}{l}\text { Human toxicological data, } \\
\text { other hazards - use and } \\
\text { accident history }\end{array}$ \\
\hline Pentachlorophenol & All formulations & Wood preservative uses & $\begin{array}{l}\text { Possible oncogenic, } \\
\text { teratogenic and fetoxic } \\
\text { effects }\end{array}$ \\
\hline $\begin{array}{l}\text { Pentachlorophenol, } \\
\text { Sodium S }\end{array}$ & All formulations & Wood preservative uses & $\begin{array}{l}\text { Possible oncogenic, } \\
\text { mutagenic and/or fetotoxic } \\
\text { effects }\end{array}$ \\
\hline Permethrin & All formulations & Agricultural crop uses & $\begin{array}{l}\text { Highly toxic to aquatic } \\
\text { organisms, oncogenicity }\end{array}$ \\
\hline Phorate & $\begin{array}{l}\text { Liquid formulations } 65 \% \\
\text { and greater (all uses); all } \\
\text { granular formulations } \\
\text { (rice) }\end{array}$ & $\begin{array}{l}\text { All uses (65\% and greater); } \\
\text { granular formulations } \\
\text { (rice) }\end{array}$ & $\begin{array}{l}\text { Acute oral and dermal } \\
\text { toxicity for granulars, } \\
\text { residue effects on avian } \\
\text { and mammalian species } \\
\text { (foliar application of liquid } \\
\text { formulation only), effects } \\
\text { on aquatic organisms }\end{array}$ \\
\hline Phostebupirim & Granular & $\begin{array}{l}\text { Corn: field, forage, pop, } \\
\text { sweet }\end{array}$ & $\begin{array}{l}\text { Toxicity to aquatic } \\
\text { invertebrates }\end{array}$ \\
\hline Picloram & $\begin{array}{l}\text { All formulations and } \\
\text { concentrations except } \\
\text { Tordon 101R }\end{array}$ & All uses & $\begin{array}{l}\text { Hazard to non-target } \\
\text { organisms (non-target } \\
\text { plants, both crop and } \\
\text { non-crop) }\end{array}$ \\
\hline Piperonyl butoxide & $\begin{array}{l}\text { Emulsifiable } \\
\text { concentrate }\end{array}$ & $\begin{array}{l}\text { Small fruits, certain berries, } \\
\text { currants }\end{array}$ & Not specified \\
\hline Profenophos & $\begin{array}{l}\text { Emulsifiable concentrate } \\
59.4 \% \text {, EPA Reg. Nos. } \\
100-599 \text { and } 100-669 \\
\end{array}$ & Cotton & Corrosive to eyes \\
\hline Pronamide & All $50 \%$ wettable & All uses & Oncogenicity \\
\hline Propanoic acid & $\begin{array}{l}\text { EAtudgrffable } \\
\text { concentrate }\end{array}$ & $\begin{array}{l}\text { Wheat, rice, edible } \\
\text { chrysanthemum, cotton, } \\
\text { clover, alfalfa, wheat-grass, } \\
\text { sideoats grama, little } \\
\text { bluestem }\end{array}$ & Not specified \\
\hline Propetamphos & $\begin{array}{l}\text { Emulsifiable concentrate } \\
50 \%\end{array}$ & Indoor domestic use & Not specified \\
\hline Pyrethrins & $\begin{array}{l}\text { Emulsifiable } \\
\text { concentrate }\end{array}$ & No uses listed & Chronic eye effects \\
\hline Resmethrin & All formulations & $\begin{array}{l}\text { Mosquito abatement and } \\
\text { pest control treatments at } \\
\text { nonagricultural sites }\end{array}$ & Acute fish toxicity \\
\hline Rotenone & $\begin{array}{l}2.5 / 5.0 \text { EC, } 5.0 \%+20.0 \% \\
\text { wettable powder }\end{array}$ & Fish toxicant & $\begin{array}{l}\text { Chronic eye and inhalation } \\
\text { effects }\end{array}$ \\
\hline
\end{tabular}


Table 1. Restricted use pesticides.

\begin{tabular}{|c|c|c|c|}
\hline $\begin{array}{l}\text { Pesticide common } \\
\text { name }\end{array}$ & Specific formulations & Specific uses & Criteria for RUP \\
\hline S-fenvalerate & $\begin{array}{l}\text { Emulsifiable } \\
\text { concentrates }(30 \%)\end{array}$ & Outdoor uses & $\begin{array}{l}\text { Possible adverse effects } \\
\text { on aquatic organisms }\end{array}$ \\
\hline Simazine & $\begin{array}{l}\text { Emulsifiable } \\
\text { concentrate }\end{array}$ & Grapes and certain berries & Not specified \\
\hline Sodium cyanide & $\begin{array}{l}\text { All capsules and ball } \\
\text { formulations }\end{array}$ & All uses & $\begin{array}{l}\text { Human inhalation hazard, } \\
\text { hazard to non-target } \\
\text { species }\end{array}$ \\
\hline Sodium dichromate & $\begin{array}{l}\text { All wood preservative } \\
\text { formulations except } \\
\text { brush-on }\end{array}$ & Wood preservative uses & $\begin{array}{l}\text { Oncogenicity, } \\
\text { mutagenicity, } \\
\text { teratogenicity and } \\
\text { fetotoxocity }\end{array}$ \\
\hline Sodium fluoroacetate & $\begin{array}{l}\text { All solutions and dry } \\
\text { baits }\end{array}$ & All uses & $\begin{array}{l}\text { Acute oral toxicity, hazard } \\
\text { to non-target organisms, } \\
\text { use and accident history }\end{array}$ \\
\hline Sodium hydroxide & Ready to use solution & $\begin{array}{l}\text { Control tree roots in sewage } \\
\text { systems }\end{array}$ & $\begin{array}{l}\text { Acute toxicity; eye, } \\
\text { inhalation and dermal } \\
\text { hazard }\end{array}$ \\
\hline $\begin{array}{l}\text { Sodium } \\
\text { methyldithiocarbamate }\end{array}$ & $32.7 \%$ anhydrous & $\begin{array}{l}\text { Soil fumigant to control } \\
\text { soilborne pests of } \\
\text { ornamental, food and fiber } \\
\text { crops and for root control in } \\
\text { sewage systems }\end{array}$ & $\begin{array}{l}\text { Dermal toxicity and } \\
\text { teratogenicity. Acute } \\
\text { toxicity of metam sodium } \\
\text { and its intended use in } \\
\text { controlling sewer root } \\
\text { growth }\end{array}$ \\
\hline Starlicide & $98 \%$ concentrates & Bird repellent & $\begin{array}{l}\text { Hazard to non-target } \\
\text { organisms }\end{array}$ \\
\hline Strychnine & $\begin{array}{l}\text { Dry baits, pellets and } \\
\text { powder formulations - } \\
\text { see specific labels }\end{array}$ & $\begin{array}{l}\text { Formulations greater than } \\
0.5 \% \text { : all uses. All } \\
\text { formulations: all uses calling } \\
\text { for burrow builders. } \\
\text { Formulations less than } \\
0.5 \% \text { : all uses except } \\
\text { below-ground hand } \\
\text { application. }\end{array}$ & $\begin{array}{l}\text { Acute oral toxicity hazard } \\
\text { to non-target avian } \\
\text { species; use and accident } \\
\text { history }\end{array}$ \\
\hline Sulfotepp & $\begin{array}{l}\text { Sprays and smoke } \\
\text { generators }\end{array}$ & All uses & $\begin{array}{l}\text { Inhalation hazard to } \\
\text { humans }\end{array}$ \\
\hline Sulfuric acid & Solution - ready to use & Potato vine desiccant & $\begin{array}{l}\text { Extremely corrosive - } \\
\text { acute toxicity to humans }\end{array}$ \\
\hline Sulfuryl fluoride & All formulations & All uses & $\begin{array}{l}\text { Acute inhalation hazard } \\
\text { and possible acute toxicity } \\
\text { hazard in humans }\end{array}$ \\
\hline Sulprofos & All formulations & All uses & Wildlife hazard \\
\hline Tefluthrin & Granular formulations & Corn grown for seed & Environmental concerns \\
\hline Terbufos & $\begin{array}{l}\text { Granular formulations } \\
15 \% \text { and greater }\end{array}$ & All uses & $\begin{array}{l}\text { Residue effects on avian } \\
\text { species; acute oral and } \\
\text { dermal toxicity }\end{array}$ \\
\hline TFM & Impregnated material & Aquatic pest control & $\begin{array}{l}\text { Complexity in use- } \\
\text { requires specialized } \\
\text { training, equipment and } \\
\text { clothing }\end{array}$ \\
\hline
\end{tabular}


Table 1. Restricted use pesticides.

\begin{tabular}{||l|l|l|l|}
\hline \hline $\begin{array}{l}\text { Pesticide common } \\
\text { name }\end{array}$ & Specific formulations & Specific uses & Criteria for RUP \\
\hline Tralomethrin & All formulations & All agricultural crop uses & $\begin{array}{l}\text { Toxicity to aquatic } \\
\text { organisms }\end{array}$ \\
\hline Tributyltin fluoride & Solution - ready to use & Antifouling paint & $\begin{array}{l}\text { Toxicity to aquatic } \\
\text { organisms including } \\
\text { shellfish }\end{array}$ \\
\hline $\begin{array}{l}\text { Tributyltin } \\
\text { methacrylate }\end{array}$ & Solution - ready to use & Antifouling paint & $\begin{array}{l}\text { Toxicity to aquatic } \\
\text { organisms }\end{array}$ \\
\hline Triisopropranolamine & $\begin{array}{l}\text { Emulsifiable } \\
\text { concentrate }\end{array}$ & All uses & $\begin{array}{l}\text { Hazard to non-target } \\
\text { organisms, specifically } \\
\text { plants both crop and } \\
\text { noncrop }\end{array}$ \\
\hline Triphenyltin hydroxide & All formulations & All uses & $\begin{array}{l}\text { Possible mutagenic } \\
\text { effects }\end{array}$ \\
\hline Zinc phosphide & $\begin{array}{l}\text { All dry formulations } 60 \% \\
\text { and greater; all bait } \\
\text { formulations; all dry } \\
\text { formulations 10\% and } \\
\text { greater }\end{array}$ & $\begin{array}{l}\text { All uses - non-domestic } \\
\text { outdoor uses (other than } \\
1-2 \% \text { formulation in/around } \\
\text { buildings); domestic uses }\end{array}$ & $\begin{array}{l}\text { Hazard to non-target } \\
\text { organisms, acute oral } \\
\text { toxicity, acute inhalation } \\
\text { toxicity }\end{array}$ \\
\hline \hline
\end{tabular}

